Denise Ribeiro Da Silva - Ana Carolina Ramos de Castro - Roseany Barros Morais Lago Frank Willam Silva Brito - Joana D'arc do Nascimento Oliveira - Vitória Silva Almeida Thomas Dalysson Costa Feitosa de Sena - Jessica Maria Santos Dias Bruno Eugênio da Costa Silva - Bruna Lorena Soares Cavalcante Sousa

\title{
FATORES DE RISCO PARA MORTALIDADE NEONATAL EM RECËM-NASCIDOS INTERNADOS NA UTI
}

$1^{\circ}$ Edição

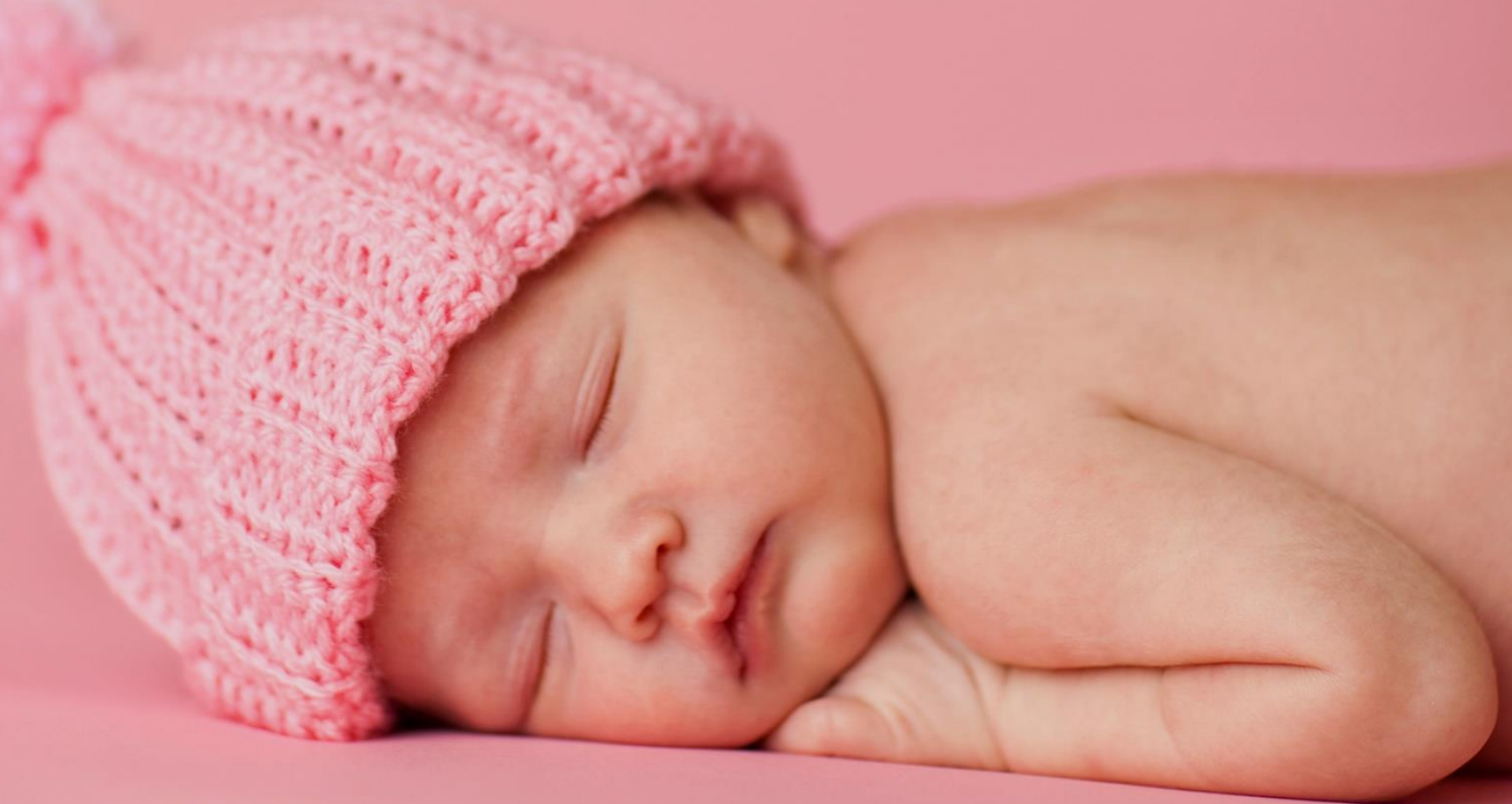

São José dos Pinhais

BRAZILIAN JOURNALS PUBLICAÇÕES DE PERIÓDICOS E EDITORA 2021 
Denise Ribeiro Da Silva - Ana Carolina Ramos de Castro

Roseany Barros Morais Lago - Frank Willam Silva Brito Joana D'arc do Nascimento Oliveira - Vitória Silva Almeida Thomas Dalysson Costa Feitosa de Sena - Jessica Maria Santos Dias Bruno Eugênio da Costa Silva - Bruna Lorena Soares Cavalcante Sousa

\section{Fatores de risco para mortalidade neonatal em recém-nascidos internados na uti}

$1^{\circ}$ Edição 


\author{
2021 by Brazilian Journals Editora \\ Copyright (C) Brazilian Journals Editora \\ Copyright do Texto (C) 2021 Os Autores \\ Copyright da Edição @ 2021 Brazilian Journals Editora \\ Diagramação: Sabrina Binotti \\ Edição de Arte: Sabrina Binotti \\ Revisão: Os autores
}

\begin{abstract}
O conteúdo dos artigos e seus dados em sua forma, correção e confiabilidade são de responsabilidade exclusiva dos autores. Permitido o download da obra e o compartilhamento desde que sejam atribuídos créditos aos autores, mas sem a possibilidade de alterá-la de nenhuma forma ou utilizá-la para fins comerciais.
\end{abstract}

\title{
Conselho Editorial:
}

Prof ${ }^{a}$. Dr ${ }^{\mathrm{a}}$. Fátima Cibele Soares - Universidade Federal do Pampa, Brasil.

Prof. Dr. Gilson Silva Filho - Centro Universitário São Camilo, Brasil.

Prof. Msc. Júlio Nonato Silva Nascimento - Instituto Federal de Educação, Ciência e Tecnologia do Pará, Brasil.

Profá. Msc. Adriana Karin Goelzer Leining - Universidade Federal do Paraná, Brasil.

Prof. Msc. Ricardo Sérgio da Silva - Universidade Federal de Pernambuco, Brasil.

Prof. Esp. Haroldo Wilson da Silva - Universidade Estadual Paulista Júlio de Mesquita Filho, Brasil.

Prof. Dr. Orlando Silvestre Fragata - Universidade Fernando Pessoa, Portugal.

Prof. Dr. Orlando Ramos do Nascimento Júnior - Universidade Estadual de Alagoas, Brasil.

Profa . Dra . Angela Maria Pires Caniato - Universidade Estadual de Maringá, Brasil.

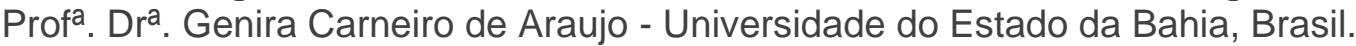

Prof. Dr. José Arilson de Souza - Universidade Federal de Rondônia, Brasil.

Prof ${ }^{a}$. Msc. Maria Elena Nascimento de Lima - Universidade do Estado do Pará, Brasil.

Prof. Caio Henrique Ungarato Fiorese - Universidade Federal do Espírito Santo, Brasil.

Prof ${ }^{a}$. Dra . Silvana Saionara Gollo - Instituto Federal de Educação, Ciência e Tecnologia do

Rio Grande do Sul, Brasil.

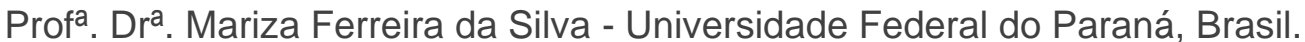

Prof. Msc. Daniel Molina Botache - Universidad del Tolima, Colômbia.

Prof. Dr. Armando Carlos de Pina Filho- Universidade Federal do Rio de Janeiro, Brasil.

Prof. Dr. Hudson do Vale de Oliveira- Instituto Federal de Educação, Ciência e Tecnologia de Roraima, Brasil.

Profa . Msc. Juliana Barbosa de Faria - Universidade Federal do Triângulo Mineiro, Brasil.

Profa ${ }^{a}$. Esp. Marília Emanuela Ferreira de Jesus - Universidade Federal da Bahia, Brasil.

Prof. Msc. Jadson Justi - Universidade Federal do Amazonas, Brasil.

Prof ${ }^{a}$. Dr ${ }^{\text {a }}$. Alexandra Ferronato Beatrici - Instituto Federal de Educação, Ciência e

Tecnologia do Rio Grande do Sul, Brasil.

Prof ${ }^{a}$. Msc. Caroline Gomes Mâcedo - Universidade Federal do Pará, Brasil.

Prof. Dr. Dilson Henrique Ramos Evangelista - Universidade Federal do Sul e Sudeste do

Pará, Brasil.

Prof. Dr. Edmilson Cesar Bortoletto - Universidade Estadual de Maringá, Brasil.

Prof. Msc. Raphael Magalhães Hoed - Instituto Federal do Norte de Minas Gerais, Brasil.

Prof ${ }^{a}$. Msc. Eulália Cristina Costa de Carvalho - Universidade Federal do Maranhão, Brasil.

Prof. Msc. Fabiano Roberto Santos de Lima - Centro Universitário Geraldo di Biase, Brasil.

Prof ${ }^{a}$. Dr ${ }^{a}$. Gabrielle de Souza Rocha - Universidade Federal Fluminense, Brasil.

Prof. Dr. Helder Antônio da Silva, Instituto Federal de Educação do Sudeste de Minas

Gerais, Brasil. 
Prof ${ }^{a}$. Esp. Lida Graciela Valenzuela de Brull - Universidad Nacional de Pilar, Paraguai.

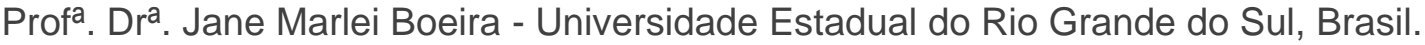
Prof ${ }^{a}$. Dra . Carolina de Castro Nadaf Leal - Universidade Estácio de Sá, Brasil.

Prof. Dr. Carlos Alberto Mendes Morais - Universidade do Vale do Rio do Sino, Brasil.

Prof. Dr. Richard Silva Martins - Instituto Federal de Educação, Ciência e Tecnologia Sul Rio Grandense, Brasil.

Prof ${ }^{a}$. Dr ${ }^{a}$. Ana Lídia Tonani Tolfo - Centro Universitário de Rio Preto, Brasil.

Prof. Dr. André Luís Ribeiro Lacerda - Universidade Federal de Mato Grosso, Brasil.

Prof. Dr. Wagner Corsino Enedino - Universidade Federal de Mato Grosso, Brasil.

Profa . Msc. Scheila Daiana Severo Hollveg - Universidade Franciscana, Brasil.

Prof. Dr. José Alberto Yemal - Universidade Paulista, Brasil.

Prof ${ }^{a}$. Dr ${ }^{\mathrm{a}}$. Adriana Estela Sanjuan Montebello - Universidade Federal de São Carlos, Brasil. Profa . Msc. Onofre Vargas Júnior - Instituto Federal de Educação, Ciência e Tecnologia Goiano, Brasil.

Prof ${ }^{a}$. Dra . Rita de Cássia da Silva Oliveira - Universidade Estadual de Ponta Grossa, Brasil. Prof ${ }^{a}$. Dr ${ }^{a}$. Leticia Dias Lima Jedlicka - Universidade Federal do Sul e Sudeste do Pará, Brasil.

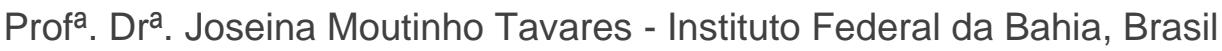

Prof. Dr. Paulo Henrique de Miranda Montenegro - Universidade Federal da Paraíba, Brasil. Prof. Dr. Claudinei de Souza Guimarães - Universidade Federal do Rio de Janeiro, Brasil.

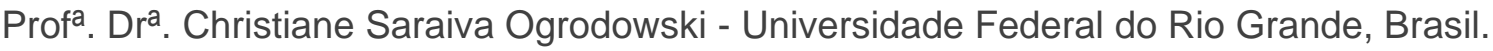
Prof ${ }^{a}$. Drª . Celeide Pereira - Universidade Tecnológica Federal do Paraná, Brasil. Prof ${ }^{a}$. Msc. Alexandra da Rocha Gomes - Centro Universitário Unifacvest, Brasil. Prof ${ }^{a}$. Dr ${ }^{a}$. Djanavia Azevêdo da Luz - Universidade Federal do Maranhão, Brasil. Prof. Dr. Eduardo Dória Silva - Universidade Federal de Pernambuco, Brasil. Profa . Msc. Juliane de Almeida Lira - Faculdade de Itaituba, Brasil.

Prof. Dr. Luiz Antonio Souza de Araujo - Universidade Federal Fluminense, Brasil.

Prof. Dr. Rafael de Almeida Schiavon - Universidade Estadual de Maringá, Brasil.

Prof ${ }^{a}$. Dr ${ }^{a}$. Rejane Marie Barbosa Davim - Universidade Federal do Rio Grande do Norte, Brasil.

Prof. Msc. Salvador Viana Gomes Junior - Universidade Potiguar, Brasil.

Prof. Dr. Caio Marcio Barros de Oliveira - Universidade Federal do Maranhão, Brasil.

Prof. Dr. Cleiseano Emanuel da Silva Paniagua - Instituto Federal de Educação, Ciência e Tecnologia de Goiás, Brasil.

Prof ${ }^{a}$. Dr ${ }^{\mathrm{a}}$. Ercilia de Stefano - Universidade Federal Fluminense, Brasil. 
Dados Internacionais de Catalogação na Publicação (CIP)

S586f Silva, Denise Ribeiro da

Fatores de Risco Para Mortalidade Neonatal em Recémnascidos Internados na Uti / Denise Ribeiro da Silva, Ana Carolina Ramos de Castro. São José dos Pinhais: Editora Brazilian Journals, 2021.

$04 \mathrm{p}$.

Formato: PDF

Requisitos de sistema: Adobe Acrobat Reader

Modo de acesso: World Wide Web

Inclui: Bibliografia

ISBN: 978-65-86230-71-0

DOI: $10.35587 /$ brj.ed.0000949

1. Pré-natal. 2. Mortalidade Neonatal. I. Silva, Denise Ribeiro da.

II. Castro, Ana Carolina Ramos de. III. Título.

CDU -615.8

Brazilian Journals Editora

São José dos Pinhais - Paraná - Brasil

www.brazilianjournals.com.br

editora@brazilianjournals.com.br 


\section{AUTORES}

Denise Ribeiro da Silva

Graduanda em Fisioterapia - Centro Universitário UNINASSAU

E-mail: denise_silva@outlook.com

Ana Carolina Ramos de Castro

Graduanda em Fisioterapia - Centro Universitário UNINASSAU

E-mail: carolcastro24281@gmail.com

Roseany Barros Morais Lago

Graduanda em Fisioterapia - Centro Universitário UNINASSAU

E-mail: roseanybarros13@gmail.com

Frank Willam Silva Brito

Graduanda em Fisioterapia - Centro Universitário UNINASSAU

E-mail: will_jd@live.com

Joana D'arc do Nascimento Oliveira

Graduanda em Fisioterapia - Centro Universitário UNINASSAU

E-mail: joanaoliveira1010@outlook.com

Vitória Silva Almeida

Graduanda em Fisioterapia - Centro Universitário UNINASSAU

E-mail: vicks415@gmail.com

Thomas Dalysson Costa Feitosa de Sena

Graduando em Fisioterapia - Centro Universitário UNINASSAU

E-mail: thomasdalysson@gmail.com

Jessica Maria Santos Dias

Graduanda em Fisioterapia - Centro Universitário UNINASSAU

E-mail: jessica18santosdias@gmail.com

Bruno Eugênio da Costa Silva

Graduando em Fisioterapia - Centro Universitário UNINASSAU

E-mail: bcostsilv@gmail.com

Bruna Lorena Soares Cavalcante Sousa

Mestre em Ciências e Saúde - UFPI

Docente do curso de Fisioterapia Centro Universitário UNINASSAU

E-mail: brunalorenasc@hotmail.com 
RESUMO: O período neonatal é determinado como os primeiros 28 dias desde o nascimento. Quadros clínicos como sepse, síndrome do desconforto respiratório, parto prematuro, baixo peso ao nascer e escore de Apgar estão entre os fatores de risco. O objetivo é descrever os principais fatores de risco para a mortalidade neonatal na Unidade de terapia intensiva neonatal. Trata-se de uma revisão integrativa da literatura, com pesquisa nas bases de dados: PUBmed, Lilacs e biblioteca eletrônica Scielo, sendo utilizados na busca os descritores combinados: Fatores de risco and pré natal; Mortalidade neonatal and Unidade de terapia intensiva neonatal e seus equivalentes no inglês. Foram selecionados artigos originais que abordaram o tema de interesse, publicados nos últimos cinco anos, língua inglesa e portuguesa, sendo disponíveis na íntegra. Na busca, encontrados 123 artigos, após análise, elegidos 10. Dentre os principais fatores de riscos encontrados nesse estudo foram, a prematuridade, baixo peso e menor Apgar. Logo, são evitáveis e podem ser prevenidos com um adequado pré-natal.

PALAVRAS-CHAVE: Fatores de risco. Pré-natal. Mortalidade neonatal precoce. Unidade de terapia intensiva neonatal. 


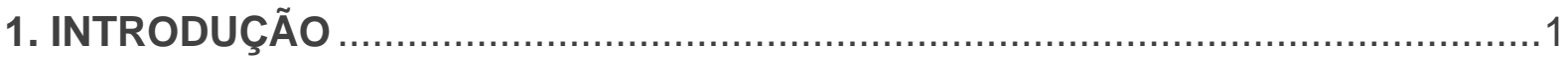

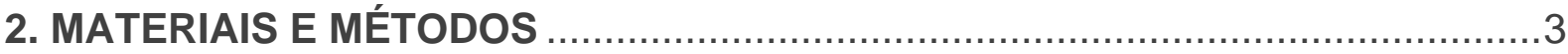

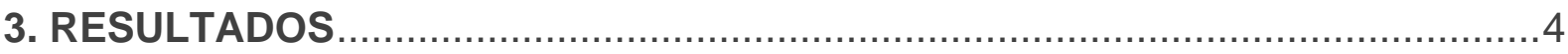

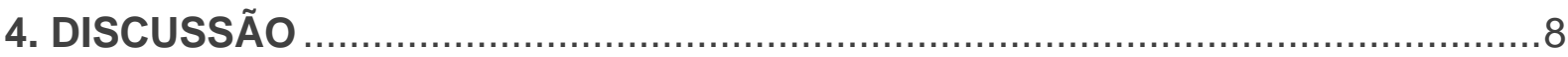

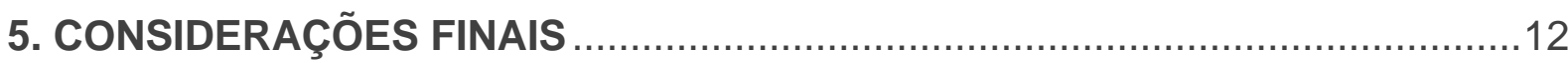




\section{INTRODUÇÃO}

O período neonatal é determinado como os primeiros 28 dias desde 0 nascimento e pode ser categorizado nos períodos neonatal muito precoce (nascimento menor a 24 horas), precoce (nascimento menor a 7 dias) e neonatal tardio, 7 dias a $<28$ dias. Neste período os óbitos representam as condições socioeconômicas, bem como as relacionadas com a qualidade da assistência prénatal, reprodutivas, durante o parto e com o recém-nascido, evidenciando lacunas na organização da rede de saúde (DEMITTO, 2017).

Evidentemente, aproximadamente $85 \%$ das mortes acontecem nos primeiros cinco anos de vida e quase metade (47\%) das menores de cinco anos ocorreu no primeiro mês de vida (SEID et al., 2019). A cobertura global de intervenções em saúde, como assistência especializada ao nascimento, imunização com toxoide tetânico, acesso a cuidados obstétricos de emergência, aleitamento materno imediato e exclusivo, prevenção de hipotermia e, se necessário, ressuscitação, cuidados com bebês com baixo peso ao nascer e tratamento de infecções, evitariam até $72 \%$ de todas as mortes de recém-nascidos, mas essas intervenções não atingem os mais necessitados (KOLOLA et al., 2016).

O coeficiente de mortalidade neonatal apresenta inúmera e complexa relação entre os fatores biológicos, sociais e de assistência à saúde. Omonitoramento da mortalidade neonatal e de seus fatores de riscos é importantepara identificar as possíveis mudanças sociais e econômicas e os possíveis progressos na qualidade dos serviços de saúde em uma população, além de ser fundamental para nortear a elaboração e implementação de novas estratégias efetivas que visem maior redução desse indicador, ainda expressivo em nosso país. (BORGES; VAYEGO, 2015).

Constata-se que muitos óbitos neonatais de causas evitáveis, normalmente está correlacionado com a falta de acesso ou qualidade de assistência durante a fase de pré-natal, seja por falta de políticas e implementação estratégicas na atenção básica, ou até mesmo falta de conhecimento da mãe quanto à realizaçãode consultas pré-natais e realização de exames nesta fase. Assim, é preciso principalmente buscar educação em saúde, pois a informação é o primeiro passo para redução dos números de $\mathrm{MN}$ por causas e fatores evitáveis (BUGES; COELHO; SILVA, 2020). 
Ressalta-se que políticas de saúde com o objetivo de qualificar a assistência obstétrica e neonatal existem, entretanto, ineficiência de planejamento, execução e avaliação das ações propostas, resulta na falta de qualidade das consultas de prénatal e da assistência ao parto, além da precariedade da estrutura física e tecnológica para o atendimento à gestante e ao bebê de alto risco, corroborando as altas taxas de mortalidade neonatal no país. (BITTENCOURT;GAíVA, 2020).

Quanto aos aspectos causais, quadro clínico como sepse, síndrome do desconforto respiratório, parto prematuro, baixo peso ao nascer, baixos escores de Apgar (medida quantitativa vitalidade neonatal), ao 1 e 5 minutos de nascimento, parto cesariana, idade neonatal na admissão e baixo nível socioeconômico, estão entre os fatores ligados aos fatores atribuíveis à mortalidade neonatal (ANDEGIORGISH et al., 2020).

Tão logo, a proposta deste estudo é a conscientização da sociedade dos direitos ao acesso a assistência especializada durante e após o parto elucidando a relevância de conhecer e entender as causas da mortalidade neonatal fundamental para a prevenção e combate, modificando os determinantes modificáveis e prevenindo os não modificáveis. Dessa forma, o objetivo é descrever os principais fatores de risco para a mortalidade neonatal na Unidade de terapia intensiva neonatal. 


\section{MATERIAIS E MÉTODOS}

O presente estudo trata-se de uma revisão integrativa de literatura, sobre os fatores de riscos que influenciam a mortalidade neonatal investigando os fatos e fenômenos com o objetivo exploratório afim de mais informações sobre o tema abordado.

A busca ocorreu entre fevereiro e junho de 2020, cuja fonte foram as bases de dados Literatura latina Americana e do Caribe (LILACS), Medical Literature Analysis and Retrievel System Online (MEDLINE/'PUBMED) e biblioteca eletrônica Scientfic Electronic Library Online (SCIELO). Todos descritores utilizados estão cadastrados nos Descritores em Ciência da Saúde (DECS), suas combinaçõesforam: Fatores de risco and pré natal; Mortalidade neonatal and Unidade de terapia intensiva neonatal, assim como equivalentes no inglês.

Os critérios de inclusão aplicados foram: artigos originais publicados no período de 2015 a 2020 com resumos de textos completos disponíveis no idioma português e inglês. Foram selecionados trabalhos pelo título, resumo e sua relevância ao trabalho com restrição ao tipo de estudo. Os critérios de exclusão foram estudos com texto completo indisponível, duplicados e que não tiveram relação com o tema proposto. 


\section{RESULTADOS}

Figura 01. Fluxograma quantitativo da busca de publicações sobre Fatores de risco, Pré-natal, Mortalidade neonatal e Uti nas principais bases de dados.
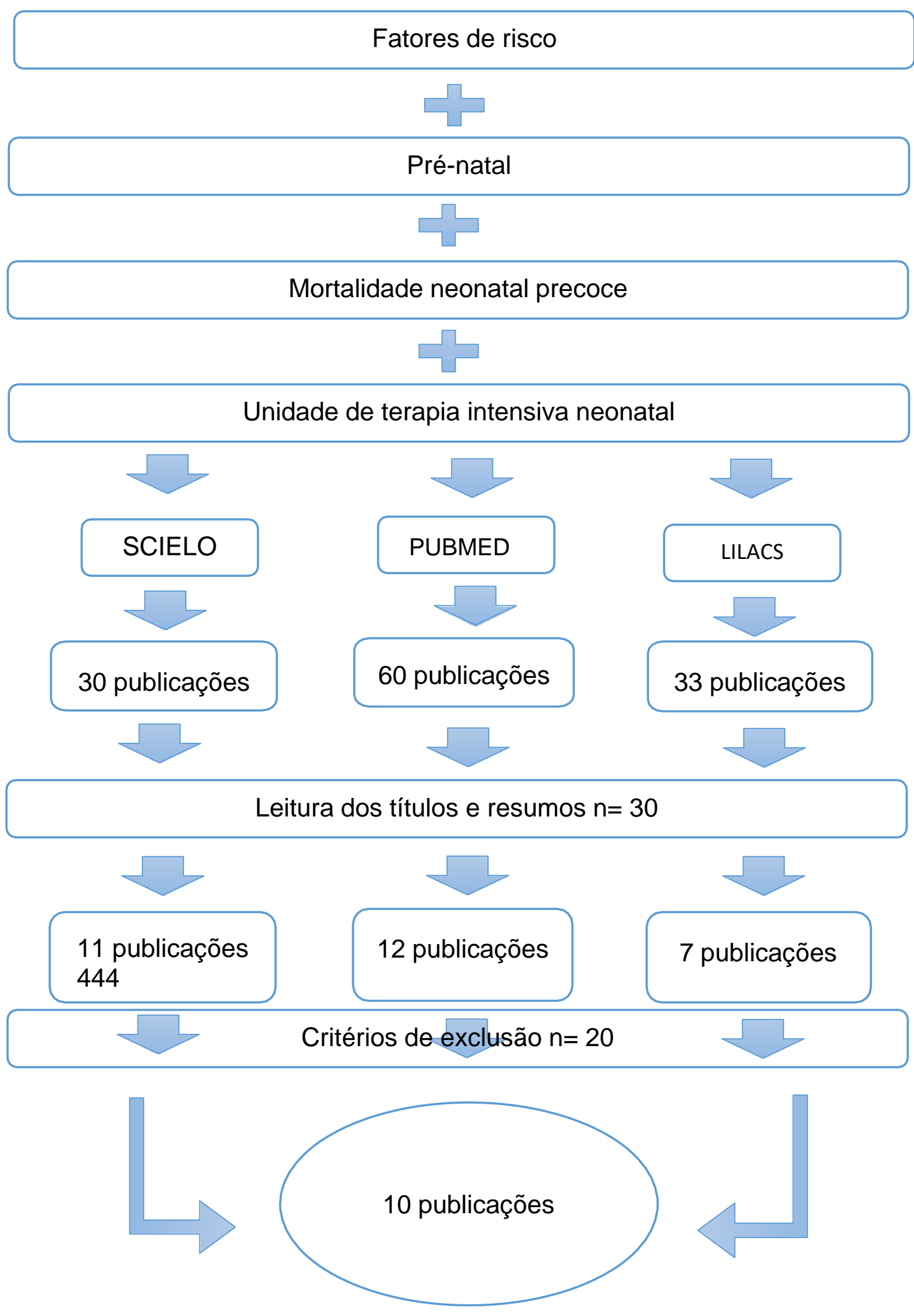
Tabela 1- Descrição de achados dos fatores de risco para mortalidade neonatal emrecém-nascidos internados na UTI.

\begin{tabular}{|c|c|c|c|c|c|}
\hline Autor/Ano & Objetivo & Tipo de Estudo & Amostra & Métodos de Avaliação & Conclusão \\
\hline $\begin{array}{l}\text { Bezerra et al., } \\
2016\end{array}$ & $\begin{array}{l}\text { Identificar os fatores } \\
\text { relacionados à mortalidade } \\
\text { neotnatal. }\end{array}$ & Transversal & 167 participantes & $\begin{array}{l}\text { Prontuários de RNs } \\
\text { nascidos vivos }\end{array}$ & $\begin{array}{l}\text { Riscos de mortalidade } \\
\text { neonatal estiveram associados } \\
\text { à qualidade da assistência ao } \\
\text { pré-natal, atendimento ao } \\
\text { parto e aos cuidados } \\
\text { recebidos pelos recém- } \\
\text { nascidos. }\end{array}$ \\
\hline $\begin{array}{l}\text { Carvalho et } \\
\text { al., } 2016\end{array}$ & $\begin{array}{l}\text { Avaliar a adequação do } \\
\text { cuidado pré-natal oferecido } \\
\text { às gestantes usuárias de } \\
\text { serviços de saúde em } \\
\text { Aracaju, estado de Sergipe, } \\
\text { Brasil, segundo a renda } \\
\text { familiar. }\end{array}$ & Transversal & 322 mulheres & $\begin{array}{l}\text { Questionário } \\
\text { estruturado }\end{array}$ & $\begin{array}{l}\text { O pré-natal em Aracaju } \\
\text { apresentou reduzido } \\
\text { percentual de adequação, } \\
\text { evidenciando a baixa } \\
\text { qualidade dos serviços, tanto } \\
\text { pelo setor público quanto } \\
\text { pelo setor privado da Saúde. }\end{array}$ \\
\hline $\begin{array}{l}\text { Santos et al., } \\
2016\end{array}$ & $\begin{array}{l}\text { Analisar a história } \\
\text { gestacional e as } \\
\text { características da } \\
\text { assistência pré-natal de } \\
\text { puérperas adolescentes e } \\
\text { adultas em uma } \\
\text { maternidade localizada em } \\
\text { uma cidade de Minas. }\end{array}$ & Descritivo & 255 puérperas & $\begin{array}{l}\text { Para a coleta de dados } \\
\text { foi utilizado um } \\
\text { instrumento } \\
\text { semiestruturado } \\
\text { contendo questões } \\
\text { relativas. }\end{array}$ & $\begin{array}{l}\text { Os resultados evidenciaram } \\
\text { a necessidade de ações } \\
\text { voltadas para a saúde da } \\
\text { mulher, evitando assim, } \\
\text { possíveis complicações que } \\
\text { terão impactos não somente } \\
\text { na vida das adolescentes. }\end{array}$ \\
\hline $\begin{array}{l}\text { Sleutjes et al., } \\
2016\end{array}$ & $\begin{array}{l}\text { Identificar os fatores de } \\
\text { risco de óbito neonatal em } \\
\text { região do interior paulista. }\end{array}$ & Caso controle & $\begin{array}{l}\text { Grupo caso=162 } \\
\text { óbitos, grupo } \\
\text { controle }=324 \\
\text { neonatos } \\
\text { nascidos. }\end{array}$ & $\begin{array}{l}\text { Fichas de óbitos } \\
\text { infantis do ano de } 2009 \\
\text { fornecidas pelos GVE e } \\
\text { do banco do SINASC. } \\
\text { Os dados foram } \\
\text { digitados no software } \\
\text { Access e analisados no } \\
\text { software estatístico. }\end{array}$ & $\begin{array}{l}\text { Fatores de risco } \\
\text { prematuridade e baixo peso } \\
\text { ao nascimento, } \\
\text { especialmente em situações } \\
\text { extremas. }\end{array}$ \\
\hline
\end{tabular}




\begin{tabular}{|c|c|c|c|c|c|}
\hline $\begin{array}{l}\text { Sousa et al., } \\
2016\end{array}$ & $\begin{array}{l}\text { Identificar os fatores } \\
\text { determinantes para o } \\
\text { nascimento de neonatos de } \\
\text { baixo peso em uma } \\
\text { maternidade pública em } \\
\text { Teresina-Pi. }\end{array}$ & Transversal & 51 mães & $\begin{array}{l}\text { Formulário com } \\
\text { questões abertas e } \\
\text { fechadas. }\end{array}$ & $\begin{array}{l}\text { A pesquisa indicou a falta do } \\
\text { companheiro, baixa } \\
\text { escolaridade, baixa renda e } \\
\text { parto cirúrgico. }\end{array}$ \\
\hline $\begin{array}{l}\text { Demisse et } \\
\text { al., } 2017\end{array}$ & $\begin{array}{l}\text { Identificar os padrões de } \\
\text { admissão neonatal e os } \\
\text { fatores associados à } \\
\text { mortalidade em neonatos } \\
\text { internados na } \\
\text { Unidade de Terapia } \\
\text { Intensiva. }\end{array}$ & Transversal & $\begin{array}{l}\text { Todos os } \\
\text { recém-nascidos } \\
\text { internados na } \\
\text { UTIN de } 1 \text { de } \\
\text { dezembro de } \\
2015 \text { a } 31 \text { de } \\
\text { agosto de } 2016 .\end{array}$ & $\begin{array}{l}\text { Questionário } \\
\text { estruturado pré- } \\
\text { testado, preparado em } \\
\text { inglês. }\end{array}$ & $\begin{array}{l}\text { Hipotermia, sepse e } \\
\text { prematuridade foram os } \\
\text { principais motivos da } \\
\text { internação na UTIN. }\end{array}$ \\
\hline $\begin{array}{l}\text { Sanders et al., } \\
2017\end{array}$ & $\begin{array}{l}\text { Analisar os fatores } \\
\text { associados à mortalidade } \\
\text { infantil, em Fortaleza, } \\
\text { Ceará. }\end{array}$ & Caso controle & $\begin{array}{l}147 \text { casos e } 441 \\
\text { controles }\end{array}$ & Formulario estruturado & $\begin{array}{l}\text { Os serviços de saúde podem } \\
\text { dar mais ênfase gestação } \\
\text { gemelar e idade gestacional } \\
\leq 36 \text { semanas, para reduzir a } \\
\text { mortalidade. }\end{array}$ \\
\hline $\begin{array}{l}\text { Alebel et al., } \\
2020\end{array}$ & $\begin{array}{l}\text { Determinar a incidência e } \\
\text { identificar os preditores de } \\
\text { mortalidade entre os } \\
\text { neonatos admitidos na } \\
\text { unidade de } \\
\text { terapia intensiva neonatal } \\
\text { do Hospital de Referência } \\
\text { Debre Markos. }\end{array}$ & Coorte & 513 neonatos & $\begin{array}{l}\text { Questionário com as } \\
\text { respctivas mães }\end{array}$ & $\begin{array}{l}\text { Mães desempregadas, não } \\
\text { iniciam a amamentação } \\
\text { exclusiva, recém-nascidos } \\
\text { admitidos por síndrome do } \\
\text { desconforto respiratório e o } \\
\text { primeiro minuto de } \\
\text { pontuação de Apgar } \\
\text { classificado como grave. }\end{array}$ \\
\hline
\end{tabular}




\begin{tabular}{|l|l|l|l|l|l|}
\hline $\begin{array}{l}\text { Desalew et al., } \\
2020\end{array}$ & $\begin{array}{l}\text { Determinar as causas e } \\
\text { preditores de mortalidade } \\
\text { neonatal em lactentes } \\
\text { internados em unidades de } \\
\text { terapia intensiva neonatal } \\
\text { nos lactentes internados em } \\
\text { unidades de terapia } \\
\text { intensiva neonatal no leste } \\
\text { da Etiópia. }\end{array}$ & Transversal & 491 neonatos & $\begin{array}{l}\text { Questionário } \\
\text { estruturado pré-testado } \\
\text { e um acompanhamento } \\
\text { lista de controle. }\end{array}$ & $\begin{array}{l}\text { As causas mais comuns de } \\
\text { morte neonatal foram parto } \\
\text { prematuro, asfixia, infecção, } \\
\text { morbidade respiratória e } \\
\text { malformação congênita. }\end{array}$ \\
\hline $\begin{array}{l}\text { Mengistu et } \\
\text { al., 2020 }\end{array}$ & $\begin{array}{l}\text { Avaliar a incidência e os } \\
\text { preditores de mortalidade } \\
\text { neonatal entre os neonatos } \\
\text { admitidos nos hospitais } \\
\text { regionais de referência de } \\
\text { Amhara, na Etiópia. }\end{array}$ & Transversal & 612 neonatos & $\begin{array}{l}\text { Entrevista pessoal e } \\
\text { revisão de prontuários, } \\
\text { utilizando questionário } \\
\text { estruturado e listas de } \\
\text { verificação. }\end{array}$ & $\begin{array}{l}\text { Idade materna avançada, } \\
\text { estado materno sem } \\
\text { instrução, status materno } \\
\text { positivo do HIV e gestações } \\
\text { múltiplas. }\end{array}$ \\
\hline
\end{tabular}

Legenda: RNs- Recém-nascidos; GVE- Grupos de Vigilância Epidemiológica; SINASC- Sistema delnformações de Nascidos Vivos; UTIN- Unidade De Terapia Intensiva Neonatal.

Fonte: As autoras. 


\section{DISCUSSÃO}

Sobretudo dentre várias condições neonatais, os três principais contribuintes para a carga global de doenças neonatais são parto prematuro, asfixia de nascimento e infecções neonatais. Os preditivos de mortalidade foramreconhecidos neste estudo por análise multivariada, incluindo hipotermia, sepse e asfixia perinatal. Informações semelhantes foram detalhados em estudos passados, apontando que os contribuidores conhecidos das mortes neonatais são problemas tratáveis e evitáveis; avaliação rigorosa e identificação de riscos são o caminho a seguir para combater e reduzir a mortalidade neonatal nos países em desenvolvimento (DEMISSE et al., 2017).

Ademais, o peso ao nascer é o fator que mais exerce influência sobre a saúde e as chances de sobrevivência infantil, quando se trata de crianças nascidas com baixo peso, em vista que os riscos de adoecer e morrer na infância são mais acentuados. As principais causas do baixo peso pode ser prevenidas por meio de acesso e qualidade do pré-natal, uma melhor nutrição materna, a eliminação do tabagismo e o tratamento oportuno de infecções (BEZERRA et al., 2016).

Entretanto, o afastamento do companheiro durante a gestação vem sendo agregado à inadequação de cuidados pré-natais; nesse estudo, ainda se observou que mulheres com menor renda apresentaram maior proporção de ausência do cônjuge. É sugerido que o companheiro presente signifique melhor apoio à mulher em seu período pré-natal, gerando resultados positivos em seu autocuidado. A maior parte das entrevistadas começaram o pré-natal no primeiro trimestre da gestação, visto que as gestantes com menor renda iniciaram o pré-natal mais tardio. teoricamente, este atraso deve-se aos problemas socioeconômicos e demográficos que dificultam o uso adequado do pré-natal (CARVALHO et al., 2016).

Uma pesquisa realizada no município de Salvador-BA, demonstrou que a proporção de óbitos foi maior entre RN de mães com menor escolaridade, sendo de unicamente $10,62 \%$ entre aqueles de mulheres com 12 ou mais anos de estudo. A maior taxa de mortalidade neonatal precoce foi encontrada entre $\mathrm{RN}$ de mães que expressaram não possuir qualquer ano de estudo, valor 20 vezes maior do que grupo de RN de mães com maior escolaridade. Averiguando as características do $\mathrm{RN}$, verificou-se que o sexo masculino em recém-nascidos demonstrou relação com o óbito neonatal, percebendo que menor mortalidade no sexo feminino seria o 
amadurecimento mais precoce do pulmão fetal no sexo feminino com redução de problemas respiratórios, que estão dentre as principais causas de óbito neonatal (SOUSA et al., 2016).

Quanto à escolaridade materna, observou-se que o tempo de estudoabaixo de quatro anos mostrou-se associado significativamente com o desfecho. No modelo inicial, verificou-se que as mães que estudaram menos de quatro anos exibiram maiores riscos de seus filhos faleceram precocemente. No entanto, após o ajuste para eliminar possíveis fatores de confusão, a mesma variável não permaneceu como um dos fatores associado ao modelo final. A baixa escolaridade materna abala de maneira forte o nível socioeconômico, a vulnerabilidade da mulhergrávida, e do recém-nascido, o acesso a serviços de saúde ,auxiliando a perpetuação de iniquidades na sobrevivência infantil, dado que essas mulheres têm menos acesso ao pré-natal ou ao cuidado com sua saúde durante a gestação (SANDERS et al., 2017).

Além do mais foram reconhecidos cinco fatores relacionados ao maior risco de óbito neonatal, dos quais são fatores intermediário como a história de óbito infantil associados ao número de consultas pré-natais e prematuridade; os demais são sujeitos de intervenções no período gestacional que também estão conectados à qualidade do cuidado neste período. Fatores de risco mais proximais foram o baixo peso ao nascer, associado à prematuridade e o baixo índice de Apgar no primeiro minuto de vida, que também está ligado à qualidade do cuidado, agora durante o trabalho de parto e o parto. Esse conhecimento indica que elevar a qualidade da atenção à saúde (no pré-natal e no parto) deve ser prioridade relacionada à prevenção do óbito neonatal (SANDERS et al., 2017).

Algumas causas de óbito no período neonatal são consideradasreduzíveis a partir de adequado acompanhamento da gestação e ao parto, e outras ainda podem ser consideradas evitáveis por meio de diagnóstico e intervenção precoces. No período pós-neonatal, as principais causas de óbito são também consideradas evitáveis e de fácil intervenção por estarem mais associadas a condições precárias de saneamento básico e de acesso aos cuidados de saúde. Nos países desenvolvidos, o componente pós-neonatal é pouco significativo, mas no Brasil apesar do decréscimo acelerado nos últimos anos, ainda há níveis elevados, eticamente inaceitáveis. Esse fato é particularmente importante quando se considera que a baixa efetividade dos serviços de assistência à saúde infantil representa um 
dos determinantes da mortalidade pós-neonatal por causas reconhecidamente evitáveis (CALDEIRA et al., 2020).

Por conseguinte, em relação aos problemas de saúde durante a gestação, a anemia, logo depois a ITU foram as complicações gestacionais mais constantes. A hipertensão arterial na gestação está ligada à risco significativamente ampliado para parto pré-termo, recém-nascido com baixo peso ao nascer e desfechos desfavoráveis, além do aumento expressivo do número de neonatos com sequelas quando sobrevivem as lesões da hipóxia cerebral. A cesariana, quando realizada de forma correta e indicada, traz benefícios para a mãe e para o bebê, no entanto, potenciais desvantagens têm sido descritas em estudos, como a maior morbimortalidade materna e morbidade neonatal (SANTOS et al., 2016).

Eventualmente, os principais grupos de causas de óbitos neonatais foram em relação à prematuridade, malformações congênitas, asfixia/hipóxia, infeções, fatores maternos e relacionados ao período gestacional e as afecções respiratórias. A prematuridade é apontada como principal causa de óbito neonatal em todas as regiões do país, definindo problemas na assistência pré-natal, no momento do parto e ao recém-nascido (BEZERRA et al., 2016).

Decerto, esse estudo verificou que os neonatos recepcionados na UTI neonatal devido à síndrome do desconforto respiratório alegavam em quantidade maior risco de morte quando comparados aos neonatos recepcionados por outras causas. Como também os recém-nascidos cujo Apgar de primeiro minuto classificado como grave alegavam maior risco de morte quando igualados àqueles cujo Apgar de primeiro minuto não foi classificado como grave (ALEBEL et al., 2020).

Precisamente o risco de mortalidade neonatal é muito maior em gestações múltiplas do que tons únicos. Esse descobrimento é apoiado por estudos anteriores realizados em várias circunstâncias. Isso pode ser decidida ao efeito de várias gestações em resultados perinatais com risco de vida. As gestações múltiplas habituam ter um risco maior de ruptura prematura de membrana, prematuridade, apresentação anormal e anormalidade do líquido amniótico, que podem aumentar a hipótese de morte neonatal (MENGISTU et al., 2020).

Em síntese, uma dimensão considerável de mortes neonatais pode ser evitada com cuidados de ressuscitação adequado. A maior parte das mortes correlacionadas à infecção pode ser evitada por meio do tratamento de infecções 
maternas durante o período gestacional, certificando um parto limpo, cuidados com o cordão umbilical e aleitamento materno exclusivo. Há uma variedade de intervenções baseados em fundamentos disponíveis que podem aperfeiçoar a sobrevida de recém-nascidos prematuros e com baixo peso ao nascer (DESALEW et al., 2020). 


\section{CONSIDERAÇÕES FINAIS}

No presente estudo verificou-se que há uma grande preocupação com as mães de baixa escolaridade, pois frente aos fatores de riscos para mortalidade neonatal estão as características sociodemográficas da mãe, considerando as principais condições clínicas críticas: a prematuridade, o baixo peso ao nascer e valores de Apgar baixo.

Tão logo, evidenciou-se a importância dos pré natais e o conhecimento sobre os fatores que possam estar relacionado a mortalidade neonatal.

Os fatores de riscos encontrados nesse estudo foram, a prematuridade, baixo peso, Apgar baixo. Esses fatores são evitáveis e podem ser prevenidos com um adequado pré-natal, informações apropriadas repassadas durante o pré-natal. Alguns fatores de risco também podem estar relacionados com a características variáveis da mãe e com as condições que elas são expostas no dia a dia. 


\section{REFERÊNCIAS}

ALEBEL, A.; WAGNEW, F.; PETRUCKA, P.; TESEMA, C.; MOGES N.A.;

ANDEGIORGISH, A.K.; ANDEMARIAM, M.; TEMESGHEN, S.; OGBAI, L.; OGBE, Z.; ZENG, L. Neonatal mortality and associated factors in the specialized neonatal care unit Asmara, Eritrea. Andegiorgish et al. BMC Public Health, v. 20, n. 10, 2020.

BEZERRA, N.F; RASSY, M.E.C; ALVES, B.L.A; CARVALHO, T.C.N; BANDEIRA, F.J.S.; Fatores relacionados à mortalidade neonatal. Rev enferm UFPE on line, $v$. 10, n. 11, p. 3951-9, nov., 2016.

BORGES, T.S.; VAYEGO, S.A. Fatores de risco para mortalidade neonatal em um município na região sul. Rev Ciência\&Saúde, v.8, n.1, p.7-14, 2015.

BUGES, N.M.; COELHO, J.R.; SILVA, N.B.A. Fatores evitáveis para mortalidade neonatal: uma revisão narrativa da literatura. Revista Amazônia Science \& Health, v. 8 , n. 1, p. 2-14, 2020.

BITTENCOURT, R.M; GAÍVA, M.A.M. Mortalidade neonatal precoce relacionado a intervenções clínicas. Rev. Bras Enferm, v.67, n.2, p.195-201, 2014.

CALDEIRA, A. P.; FRANÇA, E.; GOULART, E.M.A. Mortalidade infantil pós-neonatal e qualidade da assistência médico: um estudo caso-controle, Rev. Saúde Pública, v.39, n.1, 2020.

CARVALHO, R.A.S; SANTOS, V.S; MELO, C.M; GURGEL, R.Q; OLIVEIRA, C.C.C. Avaliação da adequação do cuidado pré-natal segundo a renda familiar em Aracaju, 2011*. Epidemiol. Serv. Saude, v.25, n.2, Apr/Jun, 2016.

DEMISSE, A.G.; ALEMU, F.; GIZAW, M.A.; TIGABU, Z. Patterns of admission and factors associated with neonatal mortality among neonates admitted to the neonatal intensive care unit of University of Gondar Hospital, Northwest Ethiopia. Pediatric Health, Medicine and Therapeutics. v. 8, p. 57-64, 2017.

DEMITTO, M.A.; GRAVENA, A.A.F.; DELL'AGNOLO, C.M.; ANTUNES, M.B.; PELLOSO, S.M. Gestação de alto risco e fatores associados ao óbito neonatal ${ }^{\star}$. Rev Esc Enferm USP, v.51, Apr., 2017.

DESALEW, A.; SINTAYEHU, Y.; TEFERI, N.; AMARE, F.; GEDA, B.; WORKU, T.; ABERA, K.; ASEFAW, A. Cause and predictors of neonatal mortality among neonates admitted to neonatal intensive care units of public hospitals in eastern Ethiopia: a facility-based prospective follow-up study. BMC Pediatrics, v.20, n.160, 2020.

KETEMA, D. B.; YISMAW, L.; MELKAMU, M.W.; HIBSTIE, Y.T; TEMESGEN, B.; BITEW, Z.W.; TADESSE, A.A; KIBRET, G.D. Neonatal mortality in the neonatal intensive care unit of Debre Markos referral hospital, Northwest Ethiopia: a prospective cohort study. BMC Pediatrics, v.20, n. 72, 2020. 
KOLOLA, T.; EKUBAY, M.; TESFA, E.; MORKA, W. Determinants of Neonatal Mortality in North Shoa Zone, Amhara Regional State, Ethiopia. PLOS ONE, v.11, n.10, 2016.

MENGISTU, B.A; YISMAW, A.E; AZENE, Z.N; MIHRET, M.S. Incidence and predictors of neonatal mortality among neonates admitted in Amhara regional state referral hospitals, Ethiopia: prospective follow up study. BMC Pediatrics, v. 20, n.142, 2020.

SANDERS, L.S.C.; PINTO, F.J.M.; MEDEIROS, C.R.B.; SAMPAIO, R.M.M.; VIANA, R.A.A.; LIMA, K.J. Mortalidade infantil: análise de fatores associados em uma capital do Nordeste brasileiro. Cad. Saúde Colet., v. 25, n. 1, p. 83-89, 2017.

SANTOS, L.A.V.; LARA, M.O.; LIMA, R.C.R.; ROCHA, A.F.; ROCHA, E.M.; GLÓRIA, J.C.R.; RIBEIRO, G.C. História gestacional e características da assistência pré-natal de puérperas adolescentes e adultas em uma maternidade do interior de Minas Gerais, Brasil. Ciência \& Saúde Coletiva, v. 23, n.2, p. 617-625, 2016.

SEID, S.S.; IBRO, S.A.; AHMED, A.A.; AKUMA, A.O.; RETA, E.Y.; HASO, T.K.; FATA, G.A. Causes and factors associated with neonatal mortality in Neonatal Intensive Care Unit (NICU) of Jimma University Medical Center, Jimma, South West Ethiopia. Pediatric Health, Medicine and Therapeutics, 2019.

SLEUTJES, F.C.M.; PARADA, C.M.G.L; CARVALHAES, M.A.B.L; TEMER, M.J. Fatores de risco de óbito neonatal em região do interior paulista, Brasil. Ciência \& Saúde Coletiva, v. 23, n. 8, p. 2713-2720, 2018.

SOUSA, A.L.G.; ROCHA, S.S.; BEZERRA, M.A.R.; ROCHA, R.C.; ARAÚJO, O.D. Fatores determinantes para o nascimento de neonatos de baixo peso internadospelo método canguru. R. Interd. v. 9, n. 1, p. 24-33, 2016. 
Agência Brasileira ISBN

ISBN: 978-65-86230-71-0 